\title{
HOMOLOGICAL INVARIANTS OF LOCAL RINGS
}

\author{
HIROSHI UEHARA
}

\section{Introduction}

In this paper $R$ is a commutative noetherian local ring with unit element 1 and $M$ is its maximal ideal. Let $K$ be the residue field $R / M$ and let $\left\{t_{1}, t_{2}\right.$, $\left.\ldots, t_{n}\right\}$ be a minimal system of generators for $M$. By a complex $R<T_{1}$, $\ldots, T_{p}>$ we mean an $R$-algebra* obtained by the adjunction of the variables $T_{1}, \ldots, T_{\rho}$ of degree 1 which kill $t_{1}, \ldots, t_{\rho}$. The main purpose of this paper is, among other things, to construct an $R$-algebra resolution of the field $K$, so that we can investigate the relationship between the homology algebra $H$ ( $R$ $\left\langle T_{1}, \ldots, T_{n}\right\rangle$ ) and the homological invariants of $R$ such as the algebra $\operatorname{Tor}^{R}(K, K)$ and the Betti numbers $B_{\rho}=\operatorname{dim}_{K} \operatorname{Tor}_{\rho}^{R}(K, K)$ of the local ring $R$. The relationship was initially studied by Serre [5]. Then Tate [6] gave the correct lower bound for the Betti numbers of a nonregular local ring. In his M. I. T. lecture (See a footnote of [6]) Eilenberg proves that

$$
B_{2}=\left(\begin{array}{c}
n \\
2
\end{array}\right)+\left(\begin{array}{c}
n \\
0
\end{array}\right) b_{1} \text { and } B_{3} \geq\left(\begin{array}{c}
n \\
3
\end{array}\right)+\left(\begin{array}{l}
n \\
1
\end{array}\right) b_{1}
$$

where $b_{1}=\operatorname{dim}_{K} H_{1}\left(R<T_{1}, \ldots, T_{n}>\right)$. In this paper these results of Eilenberg are generalized as follows :

$$
\begin{aligned}
& B_{3}=\left(\begin{array}{c}
n \\
3
\end{array}\right)+\left(\begin{array}{c}
n \\
1
\end{array}\right) b_{1}+\varepsilon_{2}, \\
& B_{4}=\left(\begin{array}{c}
n \\
4
\end{array}\right)+\left(\begin{array}{c}
n \\
2
\end{array}\right) b_{1}+\left(\begin{array}{c}
n \\
0
\end{array}\right) b_{1}^{2}-\left(\begin{array}{c}
b_{1} \\
2
\end{array}\right)+\varepsilon_{2}\left(\begin{array}{c}
n \\
1
\end{array}\right)+\varepsilon_{3}\left(\begin{array}{c}
n \\
0
\end{array}\right),
\end{aligned}
$$

and so forth, where $\varepsilon_{2}=\operatorname{dim}_{K} H_{2}(\Lambda) / H_{1}(\Lambda)^{2}, \varepsilon_{3}=\operatorname{dim}_{K} H_{3}(\Lambda) / H_{1}(\Lambda) \cdot H_{2}(\Lambda)$, and $\left.\Lambda=R<T_{1}, \ldots, T_{n}\right\rangle$. As corollaries of the above computation we obtain part of the results by Tate [6],

$$
B_{P} \geq\left(\begin{array}{c}
n \\
\rho
\end{array}\right)+\left(\begin{array}{c}
n \\
\rho-2
\end{array}\right)+\left(\begin{array}{c}
n \\
\rho-4
\end{array}\right)+\cdots, \text { for } \rho \leq 4,
$$

Received May 14, 1962.

* For definition, see a paper of Tate [6]. Throughout the paper the numbers in square brackets refer to the papers of the bibliography at the end of the paper. 
if $R$ is not regular.

If $R$ is a complete intersection, we have

$$
\begin{aligned}
& B_{3}=\left(\begin{array}{c}
n \\
3
\end{array}\right)+\left(\begin{array}{l}
n \\
1
\end{array}\right) b_{1}, \\
& B_{4}=\left(\begin{array}{c}
n \\
4
\end{array}\right)+\left(\begin{array}{c}
n \\
2
\end{array}\right) b_{1}+\left(\begin{array}{c}
n \\
0
\end{array}\right) b_{1}^{2}-\left(\begin{array}{c}
b_{1} \\
2
\end{array}\right) .
\end{aligned}
$$

$\S$ 1. The complex $R<T_{1}, \cdots, T_{p}>$

Let us consider a filtered complex $A=R<T_{1}, \ldots, T_{n}>$ with an increasing sequence of subcomplexes $R \subset R<T_{1}>\subset R<T_{1}, T_{2}>\subset \cdots \subset R<T_{1}, \ldots$, $T_{\mathrm{p}}>\subset \cdots \subset \Lambda$.

Then the graded differential algebra $A$ over $R$ (in the sequel we shall call it simply " $R$-algebra" in the sense of Tate) has the increasing filtration $\left\{R<T_{1}, \ldots, T_{p}>\right\}$ such that $R<T_{1}, \ldots, T_{p}>$ is an $R$-subalgebra. Defining $R$-modules

$$
\begin{aligned}
& D_{p, q}=H_{p+q}\left(R<T_{1}, \ldots, T_{p}>\right) \\
& E_{p, q}=H_{p+q}\left(R<T_{1}, \ldots, T_{p}>/ R<T_{1}, \ldots, T_{p-1}>\right),
\end{aligned}
$$

we have the usual exact sequence

$$
\ldots \stackrel{k}{\longrightarrow} D_{p-1, q+1} \stackrel{i}{\longrightarrow} D_{p, q} \stackrel{j}{\longrightarrow} E_{p, q} \stackrel{k}{\longrightarrow} D_{p-1, q} \stackrel{i}{\longrightarrow} \ldots
$$

for each pair $\left(R<T_{1}, \ldots, T_{p}>, R<T_{1}, \ldots, T_{p-1}>\right)$.

Thus the exact couple $C(A)=\langle D, E ; i, j, k\rangle$ is associated with $R$-algebra $\Lambda$, where

$$
D=\sum_{p, q} D_{p, q} \text { and } E=\sum_{p, q} E_{p, q}
$$

Lemma 1.1.

$$
E_{p, q} \simeq D_{p-1, q}
$$

Proof. It is sufficient to show chain equivalences $\lambda$ and $\mu$

$$
R<T_{1}, \ldots, T_{p}>/ R<T_{1}, \ldots, T_{p-1}>\underset{\mu}{\stackrel{\lambda}{\rightleftarrows}} R<T_{1}, \ldots, T_{p-1}>
$$

such that $\lambda \mu=1$ and $\mu \lambda=1$. Let $x$ be a homogeneous element of degree $p+q$ in $R<T_{1}, \ldots, T_{p}>$. Then $x=x_{1}+x_{2} \cdot T_{p}$, where $x_{1}$ and $x_{2}$ are homogeneous elements of $R<T_{1}, \ldots, T_{p-1}>$ with degrees $p+q$ and $p+q-1$ respectively. 
Obviously the residue class $\bar{x}$ is represented by $x_{2} \cdot T_{p}$. Define $\lambda(\bar{x})=x_{2}$. It is immediate to verify that $\lambda$ is well defined and is a chain mapping. Defining $\mu$ by

$$
\mu(y)=\overline{y \cdot T_{p}},
$$

we see by straightforword computation that $\lambda$ and $\mu$ are chain equivalences. This completes the proof.

By replacing the $E$-terms by the corresponding isomorphic $D$-terms, the exact couple $C(\Lambda)$ can be developed into a "lattice-like" diagram

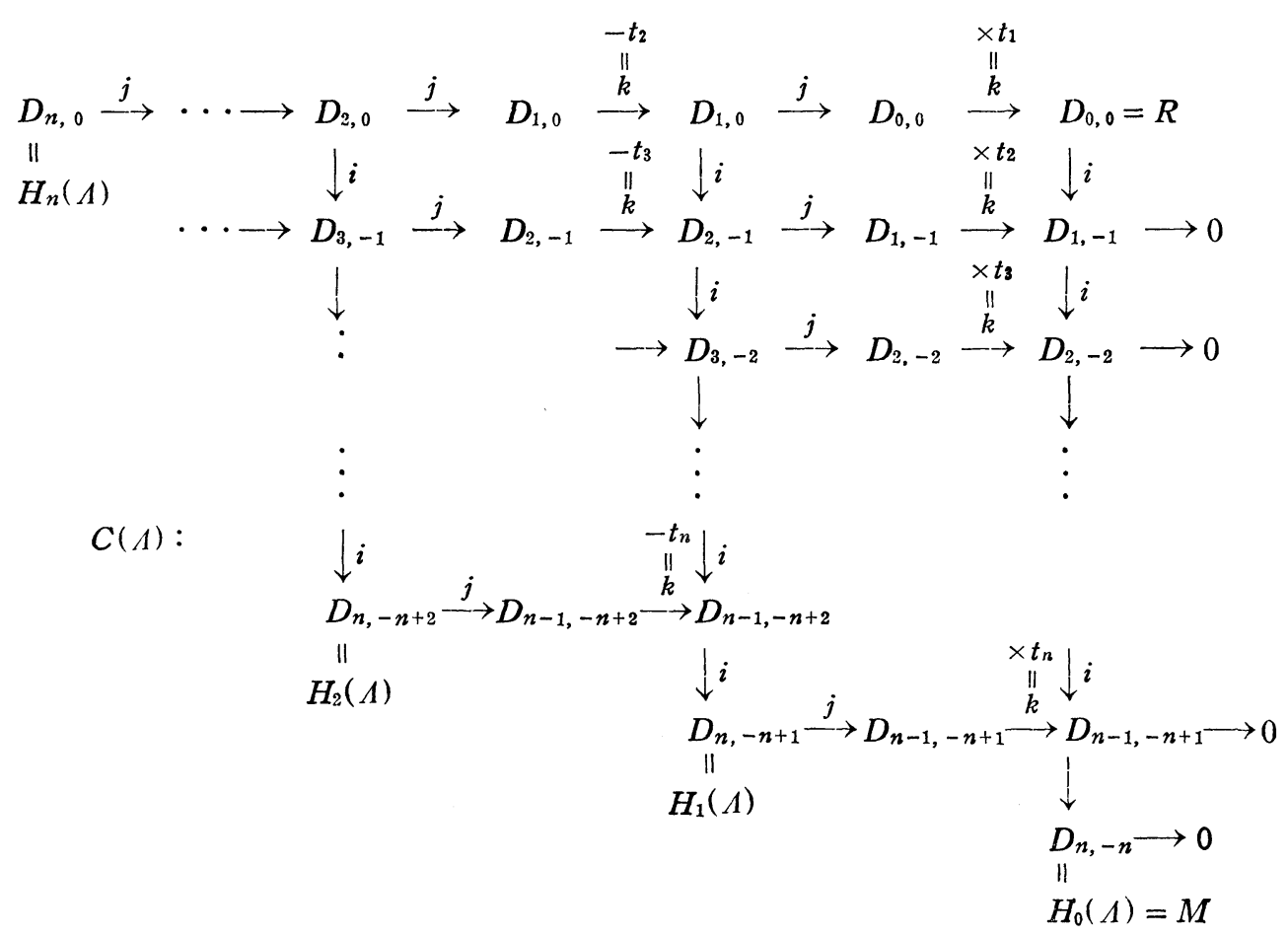

The steps from upper left to lower right are exact sequences. It is easy to see that $k_{p, q} ; D_{p, q} \longrightarrow D_{p, q}$ is the multiplication by $(-1)^{p+q} t_{p+1}$. This diagram provides us with the whole story about the following known results which have been proved by several authors [2], [6].

Proposition 1.2. The following statements are equivalent.

i) $H_{1}(\Lambda)=0$

ii) $H_{\rho}\left(R<T_{1}, \ldots, T_{p}>\right)=0$ for any $\rho \geq 1$ and for any $p(n \geq p \geq 0)$. 
iii) $\left\{t_{1}, t_{2}, \ldots, t_{n}\right\}$ is an $R$-sequence.

iv) $R$ is regular.

Proofs. i) $\rightarrow$ ii) Since $H_{1}(\Lambda)=0, k_{n-1,-n+2}$ which is the multiplication by $-t_{n}$, is onto. It follows that any element $x \in D_{n-1,-n+2}$ belongs to $\bigcap_{\rho=0}^{\infty} M^{p}$. $D_{n-1,-n+2}$. By virtue of Krull (for example see [7]) $x$ vanishes, because $D_{n-1,-n+2}=H_{1}\left(R<T_{1}, \ldots, T_{n-1}>\right)$ is a noetherian module over $R$. By the repeated use of the same argument, we can prove that $H_{1}\left(R<T_{1}, \ldots, T_{p}>\right)$ $=D_{p,-p+1}$ vanishes for all $p(n \geq p \geq 1)$. Then $i_{p,-p+2}: D_{p,-p+2} \rightarrow D_{p+1,-p+1}$ are all onto, because of the exactenss of the diagram $C(\Lambda)$. Since $D_{2,0}$ vanishes*, all $H_{2}\left(R<T_{1}, \ldots, T_{p}>\right)$ vanish. By repeating this process the proof of $\left.i\right)$ $\rightarrow$ ii) is established. ii) $\rightarrow$ iii) It is immediate by definition that $D_{p,-p}=H_{0}$ $\left(R<T_{1}, \ldots, T_{p}>\right)=R /\left(t_{1}, \ldots, t_{p}\right)$. Since $k_{p,-p}$ is isomorphic, $t_{p+1}$ is a non zero divisor for $R /\left(t_{1}, \ldots, t_{p}\right)$. This completes the proof.

iii) $\rightarrow$ iv) It is immediate by definition.

iv) $\rightarrow$ i) Without loss of generality we may assume that $\left\{t_{1}, \ldots, t_{n}\right\}$ is an $R$-sequence. Then all $k_{p,-p}$ are isomorphic so that all $i_{p,-p+1}$ are onto. Since $D_{1,0}=0$ in this case, we have $H_{1}(\Lambda)=0$.

\section{$\S 2$. Construction of a minimal algebra resolution}

Let us denote by $b_{\rho} \operatorname{dim}_{K} H_{p}(A)$ and let 1 -cycles $3_{1}^{1}, \ldots, 3 b_{1}^{1}$ represent the homology classes $Z_{1}^{1}, \ldots, Z_{b_{1}}^{1} \in H_{1}(\Lambda)$ respectively. Then by adjoining $S_{1}$, $\ldots, S_{b_{1}}$ of degree 2 which kill the cycles $3_{1}^{1}, \ldots, 3 b_{1}^{1}$ we obtain an $R$-algebra

$$
\Lambda^{(2)}=\Lambda<S_{1}, \ldots, S_{b_{1}}>; \partial_{2}^{(2)} S_{i}=3_{i}^{1}
$$

satisfying the following conditions:

a) $\Lambda^{(2)} \supset \Lambda=\Lambda^{(1)}$, and $\Lambda_{\lambda}^{(2)}=\Lambda_{\lambda}$ for $\lambda<2$,

b) $H_{1}\left(\Lambda^{(2)}\right)=0$.

Let

$$
V_{\rho}=H_{\rho}(\Lambda) /\left(H_{p-1}(\Lambda) \cdot H_{1}(\Lambda)+H_{\rho-2}(\Lambda) \cdot H_{2}(\Lambda)+\cdots+H_{\rho-\lambda}(\Lambda) \cdot H_{\lambda}(\Lambda)\right)
$$

for $\rho \geq 2$, where $\lambda=\frac{\rho}{2}$ if $\rho$ is even and $\lambda=\frac{\rho-1}{2}$ if $\rho$ is odd, and let $\varepsilon_{\rho}=\operatorname{dim}_{K} V_{\rho}$. Selecting $\rho$-cycles $3_{1}^{\circ}, \ldots, 3_{\varepsilon_{\rho}}^{\rho}$ representing the homology classes $Z_{1}^{\rho}, \ldots$, $Z_{\varepsilon_{\rho}}^{\rho} \in V_{\rho}$ and adjoining $U_{1}^{\rho+1}, \ldots, U_{\varepsilon_{\rho}}^{\rho+1}$ of degree $\rho+1$, we have an $R$-algebra

* For $t_{1}$ is a non-zero divisor for $R$. 


$$
\Lambda^{(p+1)}=\Lambda^{(\rho)}<U_{1}^{\rho+1}, \ldots, U_{\xi_{p}^{p+1}}^{p+1}>\partial_{p+1}^{(p+1)} U_{i}^{p+1}=3_{i}^{\rho}
$$

satisfying

a) $\Lambda^{(\rho+1)} \supset \Lambda^{(\rho 1}, \Lambda_{\lambda}^{(\rho+1)}=\Lambda_{\lambda}^{(\rho)}$ for $\lambda<\rho+1$

and $\Lambda_{\rho+1}^{(\rho+1)}=\Lambda_{\rho+1}^{(\rho)} \oplus R U_{1}^{\rho+1} \oplus \cdots \oplus R U_{\varepsilon_{p}}^{p+1}$

b) $H_{\rho}\left(A^{(p+1)}\right)=H_{p}\left(A^{(p)}\right) / R Z_{1}^{p}+\cdots+R Z_{\varepsilon_{p}}^{\rho}$

$$
=H_{\rho}\left(\Lambda^{(\rho)}\right) / V_{\rho}
$$

Letting $X_{p}=\Lambda_{\rho}^{(\rho)}$ and defining $\partial_{\rho+1}: X_{p+1} \rightarrow X_{\rho}$ by $\partial_{\rho+1}=\partial_{\rho+1}^{(\rho+1)}$, we obtain an $R$ algebra $X=\bigcup_{\rho} X_{p}$

$$
X: \longrightarrow X_{\mathrm{p}+1} \stackrel{\partial_{\rho+1}}{\longrightarrow} X_{\mathrm{p}} \longrightarrow \cdots \longrightarrow X_{1} \stackrel{\partial_{1}}{\longrightarrow} X_{0} \stackrel{\varepsilon}{\rightarrow} K \longrightarrow 0
$$

where $X_{0}=R$ and the mapping $\varepsilon$ is the augmentation homomorphism.

Defining vector spaces over $K, D_{p, q}=H_{p+q}\left(\Lambda^{(p)}\right)$ and $E_{p, q}=H_{p+q}\left(\Lambda^{(p)} / \Lambda^{(p-1)}\right)$, we obtain a spectral sequence

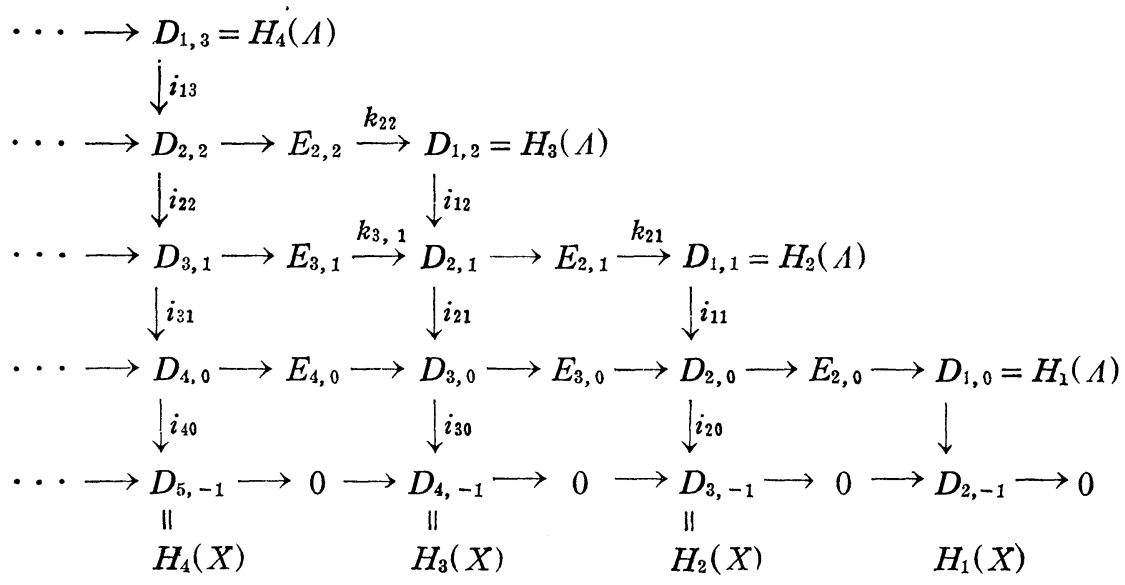

By virtue of the construction of $X$ it is seen that $D_{\rho+1,-1}=H_{\rho}\left(\Lambda^{(\rho+1)}\right)=H_{\rho}(X)$ for $\rho \geq 1, H_{1}(X)=H_{1}\left(A^{(2)}\right)=0$, and $H_{\rho}(X)=D_{\rho, 0} / V_{\rho}$. If we can prove $D_{\rho, 0} \simeq V_{\rho}$, $X$ is aspherical so that we have a desired $R$-algebra minimal resolution of $K$. In this paper we contend

Proposition 2.1.

i) $D_{2,0} \simeq H_{2}(\Lambda) / H_{1}(\Lambda)^{2}=V_{2}$,

ii) $D_{3,0} \simeq H_{3}(\Lambda) / H_{2}(\Lambda) \cdot H_{2}(\Lambda)=V_{3}$.

For the proposition we need the following two lemmas. 
Lemma 2.2.

$i_{11}, i_{21}$ and $i_{12}$ are onto.

LEMMA 2.3.

a) $k_{21}\left(E_{2,1}\right) \simeq H_{1}(\Lambda)^{2}$,

b) $k_{22}\left(E_{2,2}\right)+i_{12}^{-1} k_{31}\left(E_{3,1}\right) \simeq H_{2}(\Lambda) \cdot H_{1}(\Lambda)$.

Proof of Proposition 2.1.

It is immediate from the exactness of the spectral sequence and the above two lemmas.

Proof of Lemma 2.2.

Let $Z \in D_{2,0}$, then $Z$ is represented by a cycle

$$
3=c+\sum_{i=1}^{b_{1}} \lambda^{i} S_{i}
$$

where $c \in \Lambda_{2}$ and $\lambda^{i} \in R$. Since $0=\partial_{2} 3=\partial_{2} c+\sum_{i=1}^{b_{1}} \lambda^{i} 3_{i}^{1}$, we have $\sum_{i=1}^{b_{1}} \bar{\lambda}^{i} Z_{i}^{1}=0$ where $\bar{\lambda}^{i} \in K$. Therefore $\lambda^{i} \in M$ for all $i$. Let $\lambda^{i}=\sum_{j=1}^{n} r^{i j} \cdot t_{j}$, then

$$
\begin{aligned}
3 & =c+\sum_{i, j} r^{i j} t_{j} S_{i} \\
& =\left(c+\sum_{i, 2} r^{i j} T_{j, 3} 3_{i}^{1}\right)+\partial_{3}\left(\sum_{i, j} r^{i j} T_{j} S_{i}\right) .
\end{aligned}
$$

The cycle $3^{\prime}=\left(c+\sum_{i, j} r^{i j} T_{j} 3_{i}^{1}\right)$ represents an element $Z^{\prime} \in D_{1,1}$ whose image under $i_{11}$ is $Z$. Therefore $i_{11}$ is onto.

Secondly we wish to show that $i_{21}$ and $i_{12}$ are onto. Let $y \in X_{3}$ represent an element $Y \in D_{3,0}$. Then

$$
y=d+\sum_{j=1}^{b_{1}} \sum_{i=1}^{n} \mu^{i j}\left(T_{i} \cdot S_{j}\right)+\sum_{k=1}^{\varepsilon_{2}} \nu^{k} U_{k}^{3}
$$

where $d \in \Lambda_{3}$.

$$
0=\partial_{3} y=\sum_{j=1}^{b_{1}}\left(\sum_{i=1}^{n} \mu^{i j_{t_{i}}}\right) S_{j}+\left(\partial_{3} d-\sum_{i, j} \mu^{i j} T_{i} 3_{j}^{1}+\sum_{k} \nu^{k} \bigotimes_{k}^{2}\right) .
$$

Thus we have

$$
\sum_{i=1}^{n} \mu^{i j} t_{i}=0 \quad \text { for all } j
$$

so that $\sum_{i} \mu^{i j} T_{i}$ is 1 -cycle of $A$ and $\sum_{j}\left(\sum_{i} \mu^{i j} T_{i}\right) 3_{j}^{1}$ represents an element $Z^{\prime \prime} \in$ 
$H_{1}(\Lambda)^{2}$. From this $Z^{\prime \prime}=\sum_{k=1}^{\varepsilon_{3}} \bar{\nu}^{k} Z_{k}^{2}$, and hence $\nu^{k} \in M$. Letting $\nu^{k}=\sum_{l=1}^{n} \nu^{k l} t_{l}$ and considering $\partial_{4}\left(\sum_{k, l} \nu^{k l} T_{l} U_{k}^{3}\right)=\sum_{k} \nu^{k} U_{k}^{3}-\sum_{k, l} \nu^{k l} T_{l} 3_{k}^{2}$, we find 2-cycle of $\Lambda^{(2)}$,

$$
d+\sum_{j, i} \mu^{i j}\left(T_{i} \cdot S_{j}\right)+\sum_{k, l} \nu^{k l} T_{l} 3_{k}^{2}
$$

whose homology class $Y^{\prime}$ is mapped onto $Y$ under $i_{21}$. From the analogous argument it is easy to see that $i_{12}$ is onto. Thus the proof is omitted. This completes the proof of the Lemma.

Proof of Lemma 2.3.

Select 3-relative cycle 3 of $\Lambda^{(2)} / \Lambda$ representing an element $Z \in E_{2,1}$. Then $3=x+\sum_{i, j} \lambda^{i j} T_{i} \cdot S_{j}$, where $x \in \Lambda_{2}$ and $\sum_{i=1}^{n} \lambda^{i j} T_{i}$ is 1 -cycle of $\Lambda$. Since $k_{21}(Z)$ is represented by 2 -cycle of $\sum_{j=1}^{l_{1}}\left(\sum_{i=1}^{n} \lambda^{i j} T_{i}\right) 3_{j}^{1}$, we have $k_{21}(Z) \in H_{1}(\Lambda)^{2}$. Conversely it is obvious that $H_{1}(A)^{2} \subset k_{21}\left(E_{2,1}\right)$, beause $3_{i}^{1} 3_{j}^{1}=\partial_{3}\left(-3{ }_{i}^{1} S_{j}\right)$ for any pair $(i$, $j$ ). This completes the proof of Lemma 2.3.a).

Let $Y \in E_{3,1}$ and $y$ be 4 -relative cycle of $\Lambda^{(3)} / \Lambda^{(2)}$ representing $Y$. Then we have

$$
y=c+\sum_{i, j} \lambda^{i j} T_{i} U_{j}^{3}
$$

where $c \in \Lambda_{3}^{(2)}$ and $\sum_{i} \lambda^{i j} T_{i}$ is 1-cycle of $\Lambda$. By considering $k_{31}$ and $i_{12}, i_{12}^{-1} k_{31}(Y)$ is represented by 3 -cycle of $\Lambda, \sum_{j=1}^{\varepsilon_{2}}\left(\sum_{i=1}^{n} \lambda^{i j} T_{i}\right) 3_{j}^{2}$, whose homology class is in $H_{1}(\Lambda)$ • $V_{2} \subset H_{1}(\Lambda) \cdot H_{2}(\Lambda)$.

Let 3 be a relative 4 -cycle representing an element $Z \in E_{2,2}$, and let

$$
3=a+\sum_{b_{1} \geq k>i \geq 1} \lambda^{i k} S_{i} \cdot S_{k}+\sum_{b_{1} \geq k \geq 1} \lambda^{k k} S_{k}^{(2)}+\sum_{\substack{n \geq j \geq i \geq 1 \\ b_{1} \geq k \geq 1}} \mu^{i j k}\left(T_{i} T_{j} S_{k}\right),
$$

where $a \in \Lambda_{4}$ and $1 \cdot S_{k}^{(2)}$ is a generator of $\Lambda_{4}^{(2)}$, whose boundary is defined by $3{ }_{k} S_{k}$ (refer to [6]). Considering the boundary of 3 , we have so that

$$
\Lambda_{3} \ni \partial_{4} \mathcal{Z}=\left(\partial_{4} a+\sum_{i, j, k} \mu^{i j k}\left(T_{i} \cdot T_{j}\right) \bigotimes_{k}^{1}\right)+\sum_{k=1}^{b_{1}}\left\{\sum_{i=1}^{k} \lambda^{i k} 3_{i}^{1}+\sum_{i=k+1}^{b_{1}} \lambda^{k i} 3_{i}^{1}+\partial_{2}\left(\sum_{i, j} \mu^{i j k} T_{i} \cdot T_{j}\right)\right\} S_{k},
$$

$$
\sum_{j=1}^{k} \lambda^{i k} 3_{i}^{1}+\sum_{i=k+1}^{b_{1}} \lambda^{k i} 3_{i}^{1}+\partial_{2}\left(\sum \mu^{i j k} T_{i} \cdot T_{j}\right)=0 \text { for each } k .
$$

Therefore all $\lambda^{i k} \in M$. for any pair $(i, k)$ satisfing $b_{1} \geq k \geq i \geq 1$. Letting $\lambda^{i k}=$ $\sum_{j=1}^{n} \lambda^{i j k} t_{j}$, considering $\xi_{k}=\sum_{i=1}^{k} \sum_{j=1}^{n} \lambda^{i j k} T_{j} 3{ }^{1}+\sum_{i=k+1}^{b_{1}} \sum_{j=1}^{n} \lambda^{k j i} T_{j} 3{ }_{i}^{1}$, we obtain a 2 -cycle $\eta_{k}$ 
of $A$ by

$$
\eta_{k}-\xi_{k}=\sum_{i, j} \mu^{i j k}\left(T_{i} \cdot T_{j}\right)
$$

because $\partial_{2}\left(\xi_{k}\right)=\sum_{i=1}^{k} \lambda^{i k} 3_{i}^{1}+\sum_{i=k+1}^{b_{1}} \lambda^{k i} 3_{i}^{1}$. The straightforward computation shows $\sum_{k=1}^{b_{1}} \xi_{k} 3 \frac{1}{k}=0$, so that we have

$$
\sum_{i, j, k} \mu^{i j k}\left(T_{i} \cdot T_{j}\right) 3_{k}^{1}=\sum_{k=1}^{k_{1}} \eta_{k} 3_{k}^{1}
$$

Since $k_{22}(Z)$ is represented by $\sum_{k=1}^{l_{1} .1} \eta_{k} 3 k, k_{22}\left(E_{2,2}\right) \subset H_{2}(\Lambda) \cdot H_{1}(\Lambda)$. It is immediate to show that $H_{2}(\Lambda) \cdot H_{1}(A) \subset k_{22}\left(E_{2,2}\right)$, because $\partial_{4}\left(\eta \cdot S_{k}\right)=\eta \cdot 3 \frac{1}{k}$ for any 2 cycle $\eta$ of $\Lambda$. This completes the proof of Lemma 2.3.

\section{§3. Computation of $\boldsymbol{B}_{\rho}(\rho \leq 4)$}

Proposition 3.1.

i) $B_{1}=\left(\begin{array}{c}n \\ 1\end{array}\right) \cdot B_{2}=\left(\begin{array}{c}n \\ 2\end{array}\right)+b_{1}$,

ii) $B_{3}=\left(\begin{array}{c}n \\ 3\end{array}\right)+\left(\begin{array}{c}n \\ 1\end{array}\right) \cdot b_{1}+\varepsilon_{2}$

iii) $B_{1}=\left(\begin{array}{c}n \\ 4\end{array}\right)+\left(\begin{array}{c}n \\ 2\end{array}\right) \cdot b_{1}+\left(\begin{array}{c}n \\ 0\end{array}\right) b_{1}^{2}-\left(\begin{array}{c}b_{1} \\ 2\end{array}\right)+\left(\begin{array}{c}n \\ 1\end{array}\right) \varepsilon_{2}+\left(\begin{array}{c}n \\ 0\end{array}\right) \varepsilon_{3}$.

Proof.

In the previous section we have proved that the sequence

$$
X_{4} \stackrel{\partial_{4}}{\rightarrow} X_{3} \stackrel{\partial_{3}}{\rightarrow} X_{2} \stackrel{\partial_{2}}{\rightarrow} X_{1} \stackrel{\partial_{1}}{\rightarrow} X_{0} \stackrel{\varepsilon}{\longrightarrow} K \longrightarrow 0
$$

is exact. By definition $\operatorname{Tor}_{\rho}^{R}(K, K)$ is computed by $X_{\rho} \otimes_{K} K$ for all $\rho \leq 3$. Therefore we get i) and ii). From a general theory (for example, see [5] or [4]) we know that there exists $\widetilde{X}_{5}$ such that $\widetilde{X}_{5} \stackrel{\widetilde{\partial}^{5}}{\rightarrow} X_{4} \stackrel{\partial_{4}}{\rightarrow} X_{3}$ is exact and $\widetilde{\partial}_{\overline{5}}\left(\widetilde{X}_{5}\right) \subset$ $M X_{4}$. Therefore $B_{4}$ can be computed as stated in 3.1. iii) without knowing explicitely a system of generators for $\tilde{X}_{5}$.

Note that $\widetilde{X}_{5}$ may be considered as $X_{5}$ which we constructed in $\S 2$.

\section{$\S 4$. Corollaries and a conjecture}

Corollary 4.1 .

If $R$ is a complete intersection, we have

$$
B_{3}=\left(\begin{array}{c}
n \\
3
\end{array}\right)+\left(\begin{array}{c}
n \\
1
\end{array}\right) b_{1}
$$




$$
B_{4}=\left(\begin{array}{c}
n \\
4
\end{array}\right)+\left(\begin{array}{c}
n \\
2
\end{array}\right) b_{1}+\left(\begin{array}{c}
n \\
0
\end{array}\right) b_{1}^{2}-\left(\begin{array}{c}
b_{1} \\
2
\end{array}\right)
$$

Corollary 4.2 .

$$
B_{\rho} \geq\left(\begin{array}{c}
n \\
\rho
\end{array}\right)+\left(\begin{array}{c}
n \\
\rho-2
\end{array}\right)+\left(\begin{array}{c}
n \\
\rho-4
\end{array}\right)+\cdots
$$

for $\rho \leq 4$, if $R$ is not regular.

Proofs

By a Theorem of Assmus [1] $R$ is a local complete intersection if and only if $H(A)$ is the exterior algebra on $H_{1}(1)$. Therefore we have $\varepsilon_{2}=\varepsilon_{3}=0$ in this case. The corollary 4.1. coincides with a result of Tate [6]. The special case when $b_{1}=1 . b_{2}=b_{3}=0$, provides us with the proof of Corollary 4.2., which is the estimation of Tate [6].

Tate said in [6] that it is doubtful whether minimal $R$-algebra resolutions exist in all cases. It seems to the author that such resolution may be probable in view of the construction we consider in this paper.

\section{BIBLIOGRAPHY}

[1] E. F. Assmus, Jr., On the homology of local rings, Illinois Journal of Math., 3 (1959), 187-199.

[2] M. Auslander and D. A. Buchbaum, Codimension and multiplicity, Annals of Math., (3) 68 (1958), 625-657.

[3] H. Cartan and S. Eilenberg. Homological Algebra, Princeton University Press, 1956.

[4] D. G. Northcott, An introduction to homological Algebra, Cambridge University Press, 1960.

[ 5 ] J. P. Serre, Sur la dimension homologique des anneaux et des modules noethériens, Tokyo Symposium, 1955.

[6] J. Tate, Homology of noetherian rings and local rings, Illinois Journal of Math., 1 (1957), 14-27.

[7] O. Zariski and P. Samuel, Commutative Algebra, Vol. 1 and 2, 1958.

State University of Iowa

Iowa-City, Iowa 